\title{
Plasmonic and Semiconductor Nanoparticles Interfere with Stereolithographic 3D Printing
}

\author{
Rebecca Momper, Antonio Ibanez Landeta, Long Yang, Henry Halim, Heloise Therien-Aubin, \\ Eberhard Bodenschatz, Katharina Landfester,* and Andreas Riedinger*
}

Cite This: ACS Appl. Mater. Interfaces 2020, 12, 50834-50843

Read Online

\section{ACCESS \\ 山lll Metrics \& More \\ Article Recommendations \\ Supporting Information}

ABSTRACT: Two-photon polymerization stereolithographic three-dimensional (3D) printing is used for manufacturing a variety of structures ranging from microdevices to refractive optics. Incorporation of nanoparticles in $3 \mathrm{D}$ printing offers huge potential to create even more functional nanocomposite structures. However, this is difficult to achieve since the agglomeration of the nanoparticles can occur. Agglomeration not only leads to an uneven distribution of nanoparticles in the photoresin but also induces scattering of the excitation beam and altered absorption profiles due to interparticle coupling. Thus, it is crucial to ensure that the nanoparticles do not agglomerate during any stage of the process. To achieve noninteracting and well-dispersed nano-

Interference of nanoparticles with TPP 3D printing

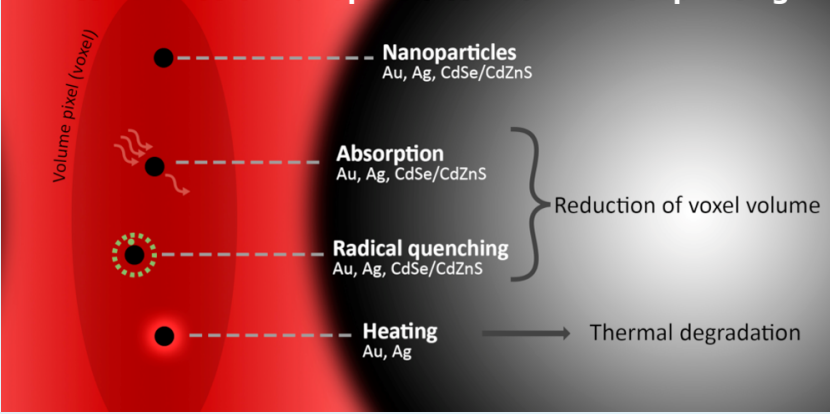
particles on the $3 \mathrm{D}$ printing process, first, the stabilization of nanoparticles in the $3 \mathrm{D}$ printing resin is indispensable. We achieve this by functionalizing the nanoparticles with surface-bound ligands that are chemically similar to the photoresin that allows increased nanoparticle loadings without inducing agglomeration. By systematically studying the effect of different nanomaterials (Au nanoparticles, Ag nanoparticles, and CdSe/CdZnS nanoplatelets) in the resin on the $3 \mathrm{D}$ printing process, we observe that both, material-specific (absorption profiles) and unspecific (radical quenching at nanoparticle surfaces) pathways co-exist by which the photopolymerization procedure is altered. This can be exploited to increase the printing resolution leading to a reduction of the minimum feature size.

KEYWORDS: 3-D printing, stereolithography, plasmonic nanoparticles, semiconductor nanoparticles, radical quenching

\section{INTRODUCTION}

Three-dimensional (3D) printing enables the quick production of complex geometries. ${ }^{1}$ Among existing 3D printing techniques, two-photon polymerization (TPP, see Scheme S1) stereolithography stands out for producing a large variety of micro- and nanosized structures, ${ }^{2}$ e.g., for photonics, ${ }^{3}$ microoptics, ${ }^{4}$ micromachines, ${ }^{5}$ biomedicine, ${ }^{6}$ or microfluidics ${ }^{7}$ applications. Here a spatially controlled fs-pulsed longwavelength (e.g., $780 \mathrm{~nm}$ ) laser is used to cross-link a photoresin via two-photon absorption to build complex polymeric structures. Due to the nonlinearity of the twophoton absorption process for curing, the resolution is improved compared to ultraviolet (UV) curing. ${ }^{8}$

Commonly used purely organic photoresins for TPP mainly ensure the good structural integrity of the printed object but lack a more specific functionality, such as optical, electrical, or magnetic activity. To further expand the library of printable, functional materials and to access even more fields of applications, like micro- and nanomachines, and (opto)electronics, the integration of different materials is a widely used method. ${ }^{1,9}$ Depending on the properties of the filler material, properties like conductivity, ${ }^{10}$ magnetism, ${ }^{11}$ or piezoelectricity, ${ }^{12}$ can be integrated. Especially, the combination of $3 \mathrm{D}$ printing and nanotechnology opens new ways toward materials with tunable properties and functionality. For example, Ceylan et al. constructed a polymeric microrobot loaded with iron oxide nanoparticles (NPs), which allow the magnetically driven motion of the swimmers due to the superparamagnetic behavior of the NPs. ${ }^{11}$

The integration of nanomaterials in a printable resin not only introduces functionality to the printed device but may also affect the printability and processability of the resin. ${ }^{13}$ Especially the stereolithographic technique, which is based on optical excitations, can be strongly affected by nanomaterials since the integrated materials can interact with the light source by absorption or scattering. Jonusauskas et al. showed this in plasmon-assisted 3D microstructuring of gold-doped poly-

Received: August 12, 2020

Accepted: October 14, 2020

Published: October 28, 2020 

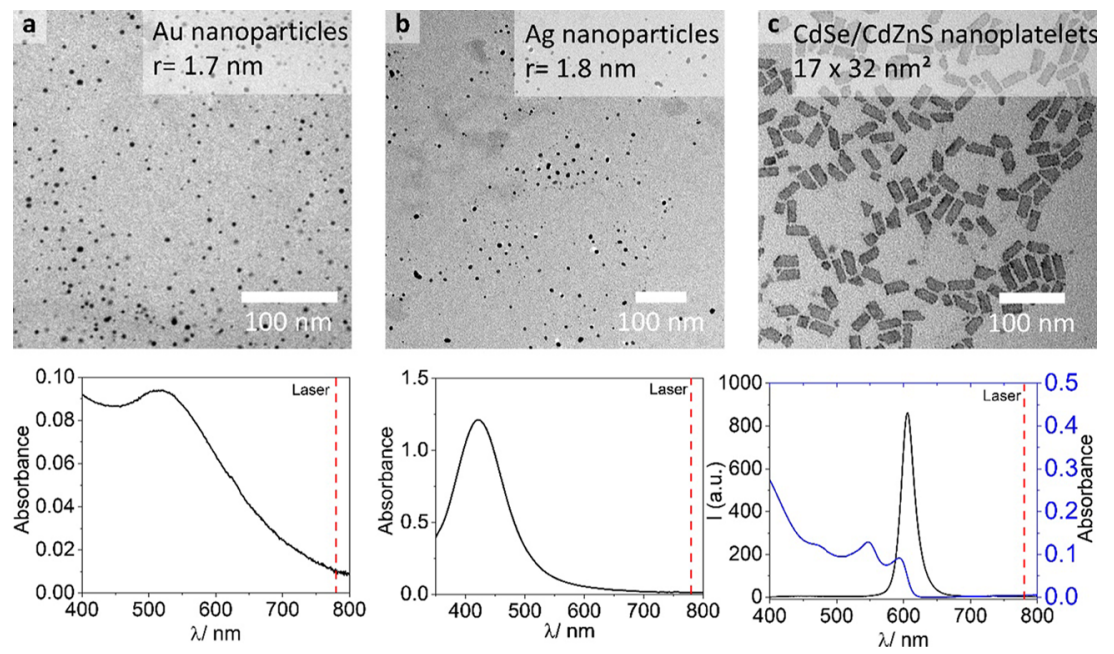

Figure 1. NPs used in this study in conjunction with a commercial acrylate-based resin for 3D printing NP/polymer composites. Upper row: transmission electron microscopy (TEM) micrographs of NPs, for higher magnification micrographs see Figure S17; lower row: corresponding absorption spectra and in case of CdSe/CdZnS NPLs the emission spectra. The dashed vertical line represents the pulsed laser wavelength at 780 nm for TPP; (a) Au NPs; (b) Ag NPs; and (c) CdSe/CdZnS NPLs.

mers. $^{14,15}$ The printed linewidth strongly depended on the concentration of Au NPs. Increasing the Au NP concentration increased the printed feature size, whereas for small concentrations the linewidth decreased. This observation led to the suggestion that Au NPs could replace the photoinitiator to some extent. The effect was explained by the plasmon effect of Au NPs. ${ }^{16,17}$ Also, for CdSe quantum dots, an improvement of the resolution and simultaneously a reduction of monomer conversion was observed by Peng et al. upon the integration of quantum dots to a photocurable resin. ${ }^{18}$ Liu et al. developed a method to produce $3 \mathrm{D}$ printed structures loaded with silver nanowires to realize electrical conductivity. ${ }^{19}$ Again, a decrease of the linewidth was observed but not further analyzed.

In TPP 3D printing, plasmonic and semiconductor NPs can interact with the light excitation either via 1 photon absorption (1PA) or 2 photon absorption (2PA, see Scheme S1) ${ }^{20-22}$ hindering the efficient formation of photogenerated radicals. ${ }^{18}$ Furthermore, NPs can also quench the polymerization directly when radical species come in contact with their surface. ${ }^{23-25}$ Both processes influence the TPP 3D printing process significantly. This is further complicated if NPs agglomerate in the photoresin. Especially, plasmonic NPs exhibit altered optical responses depending on their colloidal state, i.e., agglomeration-induced plasmonic coupling can shift the plasmon resonance substantially. The dispersion of NPs in the photoresin at high loadings without agglomeration is often a nontrivial and challenging task due to attractive forces between NPs that drive the agglomeration process. Notably, agglomeration can occur even before the printing process is started, e.g., when the NPs are introduced in the uncured photoresin. ${ }^{26,27}$ As agglomeration can influence the properties of the $\mathrm{NPs}^{26}$ (e.g., through plasmonic coupling), not only the final properties of the printed structures are affected but also interference with the printing process itself is expected. Therefore, especially agglomeration-induced scattering or absorption of the curing laser light by the agglomerates have to be considered. So far, the agglomeration of nanoparticles upon dispersion in the photoresin or upon printing has not been studied systematically. Hence, to date, it is still challenging to assess whether the previously reported effects of NPs on the 3D printing process stem from interactions of individual noninteracting NPs or from NP agglomerates with the curing laser.

For the successful printing of a resin with individual nanoparticles in a TPP 3D printing process, we first ensured the dispersibility of NPs in the uncured resin. By modifying the surface with tailor-made acrylate surfactants, we enhanced the dispersibility in the photoresin, allowing for significantly increased NP loadings compared to pristine NPs. Three different types of NPs (see Figure 1) with complementary optical properties-Au NPs (diameter $\sim 1.7 \mathrm{~nm}$ ), Ag NPs (diameter $\sim 1.8 \mathrm{~nm}$ ) and CdSe/CdZnS core/shell NPLs (17 $\mathrm{nm} \times 32 \mathrm{~nm}$ )-could be successfully used in the photoresin in volume concentrations between 0.0013 and $0.06 \mathrm{v} / \mathrm{v} \%$.

\section{RESULTS AND DISCUSSION}

Dispersion of Nanoparticles in the Uncured Resin. First, we assessed the dispersibility and colloidal stability in the uncured photoresin to allow for the homogeneous distribution of the NPs and to exclude effects caused by particle agglomerates. A commercial acrylate-based resin (IP-Dip, Nanoscribe) was used for the study. The resin consists of a mixture of multifunctional acrylates. Either the dispersion of NPs can be problematic, due to incompatible solubility of the nanoparticles' surfactants in the resin or agglomeration can occur through depletion forces induced by the oligomeric components in the photoresin. ${ }^{28}$

Pristine NPs are coated with dodecanethiol (Ag and $\mathrm{Au}$ NPs) or oleic acid (CdSe/CdZnS NPLs). To mix NPs with the photoresin different procedures are tested: (i) the NPs and the resin were both dissolved in dichloromethane (DCM) and mixed, the DCM is removed afterward, (ii) the NPs dispersed in DCM were mixed with the pure photoresin, the DCM is removed afterward, and (iii) dried NPs are mixed with undiluted photoresin. When pristine NPs are mixed with the photoresin, agglomeration occurs immediately independent of the mixing procedure. This is obvious for high loadings of NPs where agglomeration is macroscopically visible. At seemingly low loadings, particle-particle interactions could occur without being macroscopically visible. To test this in detail, we used the optical signal of Au NPs. Au NPs have distinctly 
different absorption spectra when in close contact with neighboring NPs due to plasmonic coupling and a consequent red shift and broadening of the plasmon band. ${ }^{29}$ Indeed, we observed a red shift and broadening of the absorption spectra agglomerates even for the NP-loading contents around $0.1 \mathrm{v} / \mathrm{v}$ $\%$ when Au NPs stabilized with dodecanethiol were used (see Figure 2a). This means that even though the sample seems

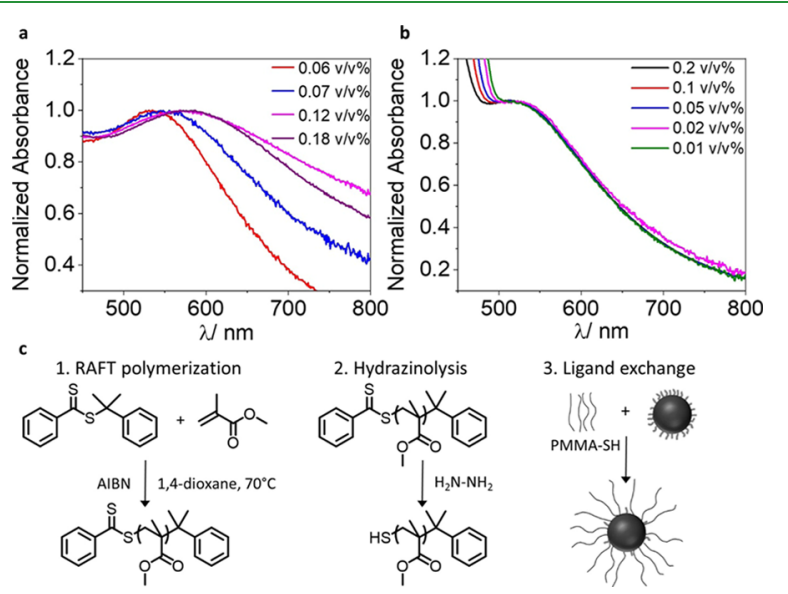

Figure 2. Absorption spectra of Au NPs stabilized with dodecanethiol (a) or PMMA-SH (b) dispersed in the IP-Dip photoresin. (a) Spectra broaden and red shift with increasing concentration, indicating the NP-concentration-dependent agglomeration tendency of Au NPs in the photoresin; (b) spectra are not shifted or broadened and no agglomeration is observed; and (c) reaction scheme of PMMA-SH synthesis and ligand exchange for particle modification.

optically clear to the eye, there is a significant particle-particle interaction and formation of agglomerates, which leads to altered optical properties of the final composite material. We note that depending on the degree of agglomeration, there should be significantly more interaction with the near infrared (NIR) laser with the Au NP-loaded photoresin. Hence, the TPP printing process will depend on the agglomeration degree of the NP in the photoresin. Since the dispersibility of the NPs depends on the solubility of the surfactant covering the NPs in the photoresin and pure dodecanethiol and oleic acid are not soluble in the photoresin, we conclude that the observed agglomeration of NPs is likely induced by the chemical incompatibility of the NP's surfactant shell with the photoresin. Having the surfactant of the same material as the photoresin should reduce the agglomeration tendency since both components, the surfactant and the photoresin, are chemically nearly identical. Thus, attractive forces between the NPs could be reduced. Indeed, the grafting of polymer chains of the same composition as the matrix is known to form a "brush" around the NPs and to decrease the attractive forces between the NPs. ${ }^{30,31}$ Therefore, to obtain a better dispersibility of the NPs in the resin by minimizing the attractive forces between particles and enhance the compatibility of the NP's surfactant shell, the particles were modified with thiol-terminated poly(methyl methacrylate) (PMMA-SH) $\left(M_{\mathrm{n}} \sim 5000 \mathrm{~g} / \mathrm{mol}\right)$ (see Methods for experimental details, see Figure S1 for the NMR spectrum). The synthesis of the polymer ligand is a two-step process, first reversible addition fragmentation chain transfer (RAFT) polymerization is used to polymerize the methyl methacrylate monomer (Figure 2c, left), and then the RAFT end group is cleaved by hydrazinolysis to obtain the thiol end group (Figure 2c, middle). Thiols are known to have a strong affinity to metals and are widely used as surfactants for metal NPs. ${ }^{32-34}$ With a ligand exchange step, the pristine surfactants (oleic acid and dodecanethiol) are replaced by the PMMA-SH surfactant (see Figure $2 \mathrm{c}$ right, and Figure S18 for thermogravimetric analysis after ligand exchange). The plasmon resonance peak of the modified Au NPs does not shift upon dispersion in the resin, indicating a lack of agglomeration (see Figure 2b). Indeed, high volume percentages up to $0.8 \mathrm{v} / \mathrm{v} \%$ are possible with no detectable agglomeration, and the loading content is only limited by an increase in viscosity, hampering the photoresin's processability. Thus, our strategy allows for significantly increased loading content and should generally be applicable to all other NP materials. Therefore, we used this method to modify Ag NPs and CdSe/CdZnS NPLs with the same polymeric surfactant and test the colloidal stability by absorption spectroscopy. Since the absorption band of $\mathrm{Ag}$ NPs overlaps with the absorption band of the resin (see Figure S2a) shifts of plasmon resonances are masked by the resin's absorption and CdSe/CdZnS NPL absorption features do not shift when agglomerated, we assess the dispersibility of Ag NPs and $\mathrm{CdSe} / \mathrm{CdZnS}$ NPLs by checking for scattering contributions $\left(1 / \lambda^{4}\right)$ in the absorption spectra (see Figure S2). In both cases, we do not observe agglomeration, highlighting the general applicability of our strategy for eliminating depletion attraction forces leading to colloidally stable NPs in the photoresin even at high loading contents. After having solved this fundamental challenge, we study the influence of wellseparated NPs embedded in the photoresin in the TPP 3D printing process.

Dispersion of Nanoparticles in 3D Printed Objects. Even if NPs are well dispersed in the uncured photoresin, the printing process could induce agglomeration or phase separation of the NPs, due to temperature gradients, viscosity changes, or shrinkage of the material. To assess this, we first estimate the diffusion rates of the NPs in the resin (see Table S2). For the following calculations, we assume continuous mode printing $(10 \mathrm{~mm} / \mathrm{s})$ and the voxel diameter to be in the order of $350 \mu \mathrm{m}$ in the $x-y$ plane (see Figure S9). With these assumptions, the dwell time of the laser per voxel is $0.03 \mathrm{~ms}$; $\mathrm{Au}$ and $\mathrm{Ag}$ NP diffusion on average $\sim 1.9 \mathrm{~nm}$ and $\mathrm{CdSe}$ / $\mathrm{CdZnS} \mathrm{NPLs} \sim 0.62 \mathrm{~nm}$ within this time frame. For the chosen volume fractions of the PMMA-SH modified NPs (between 0.02 and $0.002 \mathrm{v} / \mathrm{v} \%$ for Au NPs; between 0.013 and $0.0013 \mathrm{v} /$ v\% for Ag NPs; and between 0.06 and $0.013 \mathrm{v} / \mathrm{v} \%$ for CdSe/ $\mathrm{CdZnS} \mathrm{NPLs)} \mathrm{the} \mathrm{average} \mathrm{center-to-center} \mathrm{interparticle}$ distance is between 45 and $125 \mathrm{~nm}$ for $\mathrm{Au}$ and $\mathrm{Ag}$ NPs and 250-435 nm for CdSe/CdZnS NPLs (see Figure S8). We further analyzed the composition of the pure photoresin by NMR spectroscopy (see Figure S11) and gel permeation chromatography (GPC, see Figure S10). ${ }^{1} \mathrm{H}$ NMR spectroscopy revealed that the resin is composed mainly of multifunctional acrylates for cross-linking (signals between 4 and $6.5 \mathrm{ppm}$ ) and that the photoinitiator (signals between 8 and $6.75 \mathrm{ppm}$ ) is present in high concentrations. This was further confirmed by GPC analysis, which shows only oligomeric macromolecules with a maximum molecular weight of $\sim 1200 \mathrm{~g} / \mathrm{mol}$. This lets us conclude that TPP printing should lead to a high cross-linking density and little chaingrowth toward higher molecular weight polymers. Due to the short dwell time, the resulting short diffusion distance and the high concentration of initiator molecules (see Figure S11), we thus expect that the NPs should be trapped in the resin by the 

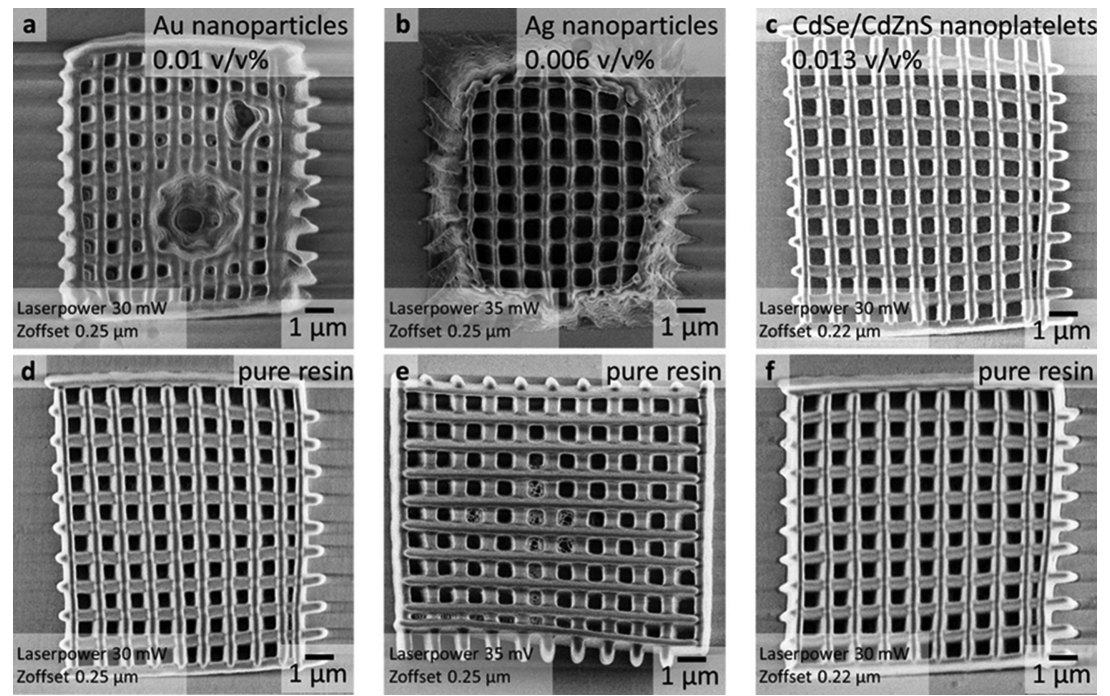

Figure 3. Scanning electron microscopy (SEM) micrographs of 3D printed woodpile structures with and without integrated NPs. (a) For the woodpile structure loaded with Au NPs, a strong tendency for thermal degradation in comparison to pure resin at the same printing parameters (d) is observable; (b) for the woodpile structure loaded with Ag NPs, polymerization and cross-linking are insufficient in comparison to pure resin (e); (c) woodpile structure loaded with $\mathrm{CdSe} / \mathrm{CdZnS}$ nanoplatelets print nearly indistinguishably compared to the pure resin (f).

cross-linking before they can agglomerate to exerted attractive forces induced by the printing process.

To prove these assumptions, we assess the distribution of the PMMA-SH modified NPs in 3D printed pyramids $(10 \mu \mathrm{m}$ $\times 10 \mu \mathrm{m} \times 5 \mu \mathrm{m})$. After printing using a commercial TPP 3D printer (Photonic Professional GT2 photonic, Nanoscribe), we used a focused ion beam (FIB) to prepare a thin lamella $(\sim 100$ $\mathrm{nm}$ ) of the 3D printed pyramids (see Figure S12) embedded in a Pd matrix. We consecutively analyzed these lamellae using transmission electron microscopy (TEM). In all cases, the NPs ( $\mathrm{Au}, \mathrm{Ag}$, and $\mathrm{CdSe} / \mathrm{CdZnS}$ ) are well separated and stochastically distributed inside the cured resin (see Figures S13-S16 for EDX). Since we know the thickness of our lamella and the visible area in our TEM micrographs, we could estimate the observable volume. Thus, by determining the number of NPs per TEM image we can estimate the volume concentration of the NPs integrated into the TPP 3D-printed resin (see the Supporting Information, Table S3). For all NP/ polymer composites, the NP concentration estimated by TEM is in good agreement with the initial concentration in the photoresin. Thus, we can conclude that the printing process with PMMA-SH modified NPs does not lead to agglomeration or phase separation, e.g., at the surface of the printed structures. After having confirmed the colloidal stability of NPs in the photoresin and their stochastic distribution in the 3D-printed structure, we now discuss how the presence of the different types of NPs influences the printing process via absorption and radical quenching processes.

Effects of Nanoparticles on Stereolithographic TPP 3D Printing. In stereolithographic TPP 3D-printing, solidification of the photoresin is based on radical polymerization and the cross-linking of, e.g., acrylate-based monomers and oligomers. ${ }^{35}$ Using a pulsed laser, the photoinitiator is activated via $2 \mathrm{PA}$ and radicals are formed allowing for radical polymerization and cross-linking. For sufficient laser power (LP), the generated radical concentration is sufficiently high and the resin is polymerized. On the one hand, if the absorbed energy in the volume where the polymerization is to occur, the so-called voxel (volume pixel), is too high, thermal degradation can occur. On the other hand, if the energy input in the voxel is too low, the polymerization does not start or is terminated prematurely, e.g., due to termination by dissolved oxygen.

By the integration of the NPs, the energy density can be affected in various ways. NPs can absorb the laser excitation either directly via 1PA (Au NPs) or via 2PA (Au and Ag NPs, and $\mathrm{CdSe} / \mathrm{CdZnS} \mathrm{NPLs).} \mathrm{Upon} \mathrm{excitation,} \mathrm{plasmonic} \mathrm{NPs}$ dissipate the absorbed energy in the form of heat, CdSe/ CdZnS NPLs predominantly by the emission of photons. While both processes decrease the available photon flux in the voxel for activation of the initiator, the influence on the TPP $3 \mathrm{D}$ printing process is expected to be distinctly different. Heat dissipation can cause additional thermal degradation of the organic materials in the proximity of plasmonic NPs, while energy dissipation via fluorescence from semiconductor NPs such as $\mathrm{CdSe} / \mathrm{CdZnS}$ should not hamper the integrity of the polymeric matrix material. Additionally, the large surface-tovolume ratio of NPs can lead to direct radical quenching when the radical-bearing (macro)molecules are in close proximity to the NPs' surfaces. To investigate these effects on the stereolithographic TPP 3D printing process, we printed and compared woodpile structures $(10 \mu \mathrm{m} \times 10 \mu \mathrm{m} ; 1 \mu \mathrm{m}$ spacing, 10 layers) with and without NPs at various laser powers and $\mathrm{z}$ offsets (see Figure 3 and Figure S3). The z-offset determines the shift of the focal point along the surface normal. Within the tested parameter space, three regions can be identified: (i) the material is not or not well polymerized, (ii) the material shows a good printing quality, and (iii) thermal degradation of the material (see Figure S3). As expected, for the pure photoresin, a large variety of parameters allow for printing with good quality. Only at the highest laser power a tendency to thermal degradation of the material is observable. In the presence of $\mathrm{Au}$ NPs, a pronounced thermal decomposition is observed even for a relatively low laser power (see Figures 3a,d and S3). Only a small laser power and a small z-offset lead to a good printing result. In comparison to Au NP/photoresin composites, in the presence of Ag NPs, much higher laser powers are required to obtain a good printing result. Lower laser powers lead to incomplete polymerization and cross-linking (see Figure 3b,e). 
In clear contrast to the plasmonic NPs, the integration of semiconductor CdSe/CdZnS NPLs does not change the useful printing parameter space significantly (see Figures $3 c, f$ and S3). Hence, in summary of the above, plasmonic NPs require high laser powers to obtain fully polymerized structures, however, high laser powers could lead to thermal degradation. Thus, for a given laser power, only a small amount of plasmonic NPs in the photoresin will allow sufficient polymerization without thermal degradation. For semiconductor NPs the thermal degradation is less problematic since they dissipate absorbed light via fluorescence. This shows that the type of material embedded in the photoresin is important not only in terms of direct interaction with the excitation beam, which alters the voxel volume, but the secondary effects after excitation similarly play a crucial role and must be considered.

To characterize the effect of PMMA-SH stabilized NPs on the printing process more in detail, we analyzed the linewidth of the woodpile structures (see Figure 4). In all cases, the
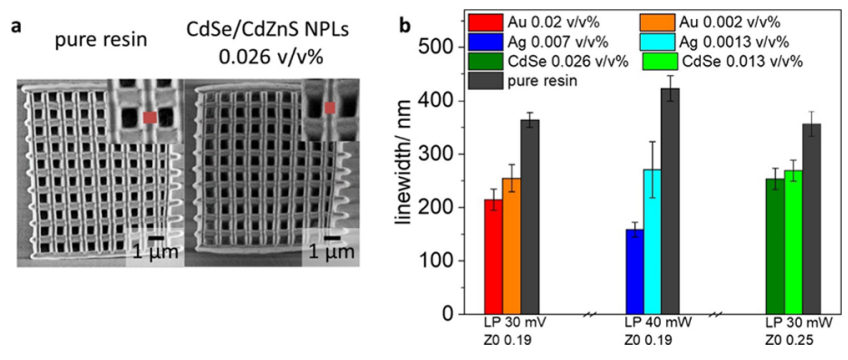

Figure 4. Decrease of the linewidth in the presence of NPs. (a) SEM micrograph of woodpile structures in the absence and the presence of $\mathrm{CdSe} / \mathrm{CdZnS}$ NPLs (0.026 v/v\%); (b) measured linewidths with all types of NPs at various concentrations.

presence of NPs decreases the linewidth significantly (28$62 \%)$. Furthermore, for all NPs increasing the NP concentration decreases the linewidth in a concentration-dependent manner (up to a $62 \%$ decrease). Notably, Ag NPs lead to the strongest effect. Here, even for relatively low volume fractions $(0.007 \mathrm{v} / \mathrm{v} \%)$, the decrease in linewidth of up to $62 \%$ is observed, whereas for the highest Au NPs (0.02 v/v\%) and $\mathrm{CdSe} / \mathrm{CdZnS}$ NPL concentrations $(0.026 \mathrm{v} / \mathrm{v} \%)$, the maximum decrease is 41 and $28 \%$, respectively.

Interference of NPs with the Stereolithographic TPP 3D Printing Process. Depending on the NP material, we observed different onsets of effects on the printing. The main observations are (i) earlier thermal degradation of the resin in the presence of $\mathrm{Au}$ NPs, (ii) higher laser power required for the polymerization of the resin in the presence of Ag NPs in comparison to all other NP-loaded photoresins, and (iii) a general concentration-dependent tendency of decreasing the linewidth of the woodpile structures in the presence of all studied NPs. The NPs can mainly cause three different processes, which all affect the $3 \mathrm{D}$ printing process (see Scheme 1).

The NPs can absorb light, either by 2PA (mainly in or near the focal volume where the photon flux is sufficiently high. This happens for plasmonic $\mathrm{NPs}^{20,22}$ and $\mathrm{CdSe} / \mathrm{CdZnS}$ $\mathrm{NPLs}^{21}$ ) or 1PA if there is a direct spectral overlap of the NP absorption profile with the laser line (in the whole pathway of the laser beam). In our case, only Au NPs can undergo 1PA (see Figure 1 for absorption spectra, the red dashed line indicates the laser wavelength). For both types of absorption
Scheme 1. Interference Pathways of NPs with the TPP 3D Printing Process ${ }^{a}$

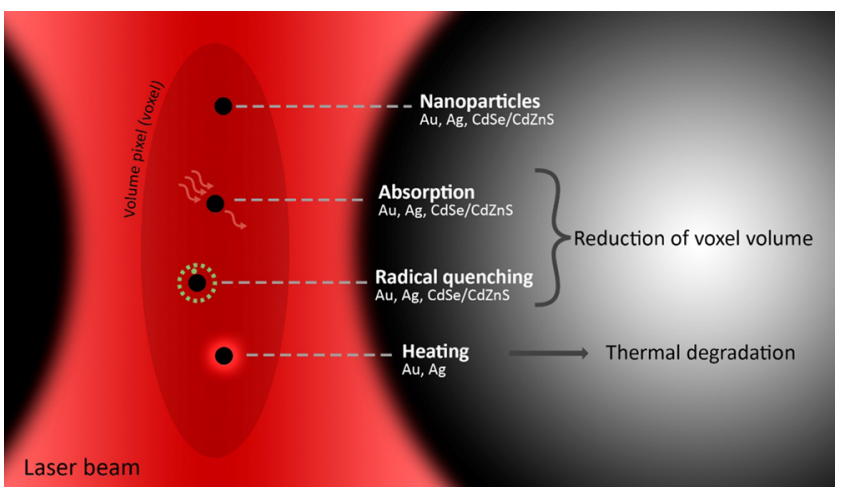

${ }^{a} \mathrm{NPs}$ can absorb the laser light either via $1 \mathrm{PA}$ or $2 \mathrm{PA}$, quench radicals created by the photoinitiator, and dissipation of the absorbed energy in the form of heat. Both absorption of laser light and quenching of radicals lead to a reduction of the voxel volume, while heating leads to thermal degradation of the material.

pathways, the absorption of light reduces the photon flux in the focal volume and therefore fewer radicals are generated. Thus, the voxel volume shrinks with increasing NP concentrations because the photon flux is not high enough to generate sufficient concentrations of radicals over a larger volume for radical polymerization. This directly leads to a reduction of the minimum printable linewidth while at the same time higher laser powers are required to generate enough radicals. Moreover, the absorption of light can induce heating of the $\mathrm{Au}$ and $\mathrm{Ag} \mathrm{NPs.}{ }^{36}$ This explains the strong thermal decomposition of the resin in the presence of Au NPs since they absorb the laser light predominantly via direct 1PA. For $\mathrm{Ag}$ NPs the thermal decomposition effect is less prominent because fewer photons are absorbed since 2PA is a nonlinear effect that requires a high density of coherent photons (as found in the focal point, but not further away from the focus). Similarly, the semiconductor $\mathrm{CdSe} / \mathrm{CdZnS}$ NPL-loaded photoresin leads to a reduction in the voxel volume due to 2PA in the focal spot. However, the energy is dissipated as fluorescence and thermal decomposition is mitigated. While these findings can explain our observation regarding the concentration-dependent reduction of the minimal printable feature size and the tendency for thermal decomposition of some NPs, other material specific effects play a similarly important role.

Besides the absorption of light, also direct interaction of the NPs with the radicals in their proximity is possible. ${ }^{23-25}$ Indeed, radicals can be quenched simply by the presence of NPs. Thus, this effect can also reduce the voxel volume. This could be material specific in our case since for the three different NPs we observe different extents of printing linewidth reduction. We observe the strongest effect for Ag NPs and the weakest effect for $\mathrm{CdSe} / \mathrm{CdZnS}$ NPLs. To analyze the quenching effect decoupled from the 1PA or 2PA absorption effect described above, we assessed the degree of conversion of methacrylate monomers by the conversion of the double bond in the NP-loaded photoresins relative to the pure resin under direct UV-light curing by Fourier transform infrared spectroscopy (FTIR, see the Supporting Information, Figures S4-S7). Here, the absorption of NPs does not hamper the photopolymerization significantly because the photoinitiators (and 
the NPs present) are excited homogeneously throughout the sample directly via $1 \mathrm{PA}$ at high intensities. Thus, even in the presence of NPs there is always sufficient UV-light to excite the photoinitiator. Vibrational bands associated with $\mathrm{CH}_{2}=\mathrm{CH}$, $\mathrm{C}=\mathrm{O}$, and $\mathrm{C}-\mathrm{O}$ groups, ${ }^{37}$ could be assigned in all spectra. All spectra were normalized to the $\mathrm{C}=\mathrm{O}$ peak (stemming from the carbonyl groups in the acrylate resin), since this signal remains constant during the polymerization reaction. The intensity of the bands assigned to the double bond decreases upon reaction (see Figure 5a). The degree of relative

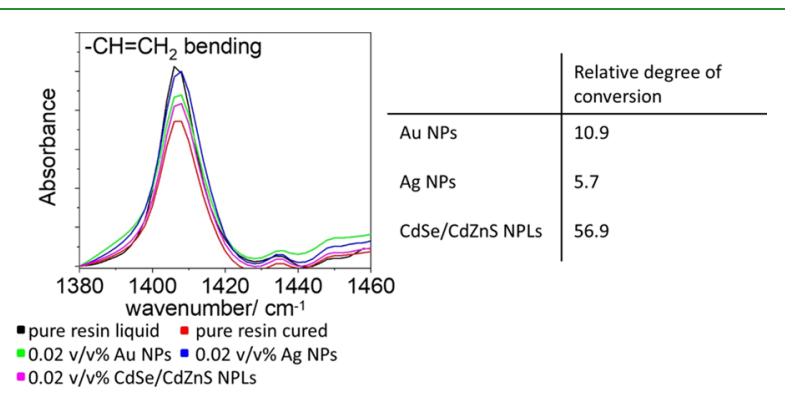

Figure 5. Fourier transform infrared (FTIR) spectra of the cured and uncured resin with and without NPs. By comparing the integrated peak intensities of the $\mathrm{C}=\mathrm{O}$ stretching and the $\mathrm{C}=\mathrm{C}$ bending mode, the relative degree of conversion is determined.

conversion was calculated by comparing the $-\mathrm{CH}=\mathrm{CH}_{2}$ bending mode band to the $\mathrm{C}=\mathrm{O}$ band before and after $\mathrm{UV}$ curing for the NP-loaded photoresins compared to the pure resin $^{37}$ (see Figure $5 b$ and Table S1). The relative degree of conversion under UV-light is the highest for the resin loaded with CdSe/CdZnS NPLs (57\%), followed by Au NP (11\%) and Ag NP-loaded resins (6\%). This finding is well in line with our observed trends of linewidth reduction as a function of the NP material. Our experiment shows that Ag NPs have the strongest direct radical quenching effect, followed by Au NPs and $\mathrm{CdSe} / \mathrm{CdZnS} \mathrm{NPLs.}$

In addition to the different absorption behaviors and the quenching properties, the difference in the interparticle distance at a given volume fraction of NPs in the resin must also be taken into account. Since the size of the used NPs are different, the interparticle distance varied for the same volume concentration (see Figure S9). For the same volume concentration $(0.013 \mathrm{v} / \mathrm{v} \%)$, the interparticle distance of $\mathrm{CdSe} / \mathrm{CdZnS}$ NPLs $(436 \mathrm{~nm})$ is much larger compared to $\mathrm{Au}$ NPs or Ag NPs (53 and $56 \mathrm{~nm}$ ) (see Figure S8). Hence, the number of NPs in the voxel volume of CdSe/CdZnS NPLs is around 10 times smaller than for Au NPs and Ag NPs (see Figure S9) and the observed direct radical quenching effects are expected to be less prominent.

\section{CONCLUSIONS}

For the integration of plasmonic and semiconductor NPs in the stereolithographic 3D printing, we could successfully show how NPs can be stabilized in the uncured photoresin and how this stability can be maintained throughout the printing process. For stabilizing the NPs in the resin, a PMMA-SH polymer was used as the surfactant. Following this approach, all NPs studied could be dispersed without agglomeration in the uncured resin. Importantly, the colloidal stability was preserved throughout the printing process: the NPs do not agglomerate or phase separate since the cross-linking is much faster than the diffusion rate of the NPs, and therefore the NPs are trapped as isolated particles within the cured resin. Being certain that individual NPs and not agglomerates are printed in the $\mathrm{NP} /$ photoresin composites allowed us to study the influence of the NP material's properties on the TPP printing process in a concentration-dependent manner. For this, printed test structures were analyzed for their minimum feature size and three main conclusions can be drawn: (i) in the presence of Au NPs, strong thermal decomposition was observed due to their 1PA; (ii) in the presence of Ag NPs, the heating effect was less pronounced since Ag NPs can only absorb laser light via $2 \mathrm{PA}$, but at the same time $\mathrm{Ag} \mathrm{NPs}$ exhibit the highest tendency for direct radical quenching; (iii) the decrease of the linewidth for CdSe/CdZnS NPLs is to a large extent due to 2PA, mitigating at the same time thermal degradation due to dissipation of energy in the form of photoluminescence. This fundamental understanding of how to integrate NPs in the resin and how NPs interfere the printing must be considered when creating NP-enriched, functional polymeric structures via TPP 3D printing. Furthermore, our findings allow for increasing the printing resolution by adding NPs, leading to a reduction of the minimum feature size. Our results will allow for finer 3D-printed refractive optics utilizing the properties of the embedded noninteracting nanoparticles.

\section{METHODS}

Characterization. For optical spectroscopy, NP/NPL dispersions were transferred to a quartz glass cuvette $(10 \mathrm{~mm})$. The NP-loaded uncured resins were deposited on a glass slide and covered with a glass coverslip. Absorption spectra were recorded on a Cary 60 spectrophotometer. Photoluminescence spectra (for NPL dispersions) were recorded using a Prizmatix Silver high-power lightemitting diode (LED) (emission peak $369 \mathrm{~nm}$ ) for excitation and an Avantes SensLine AvaSpec-HSC-TEC for data collection.

To analyze the printed structures using TEM, the samples were coated with a Pd layer and a thin lamella was prepared using a focused ion beam (FIB) system (FEI Nova 600 NanoLab FIB). Transmission electron microscopy was performed using a JEOL1400 or Tecnai FEI 20 microscope (acceleration voltage 120 and $200 \mathrm{kV}$, respectively). EDX spectra were recorded using an EDAX detector unit. The samples were deposited on carbon-coated copper grids ( 400 mesh) by drop-casting or using the focused ion beam. Scanning electron microscopy was performed on a Hitachi SU8000 with an acceleration voltage between 0.100 and $0.187 \mathrm{kV}$.

${ }^{1} \mathrm{H}$ NMR spectra were recorded with a Bruker Avance spectrometer operating at $300 \mathrm{MHz}$. The molecular mass of the IP-DIP resin was determined by GPC using a PSS SECcurity device (Agilent Technologies 1260 Infinity). The resin was dissolved in tetrahydrofuran (THF) and a calibration curve for PMMA in THF was applied.

ICP-OES measurements were performed with an ACTIVA M spectrometer (Horiba Jobin Yvon, Bernsheim, Germany) equipped with a Meinhardt-type nebulizer, a cyclone chamber, and controlled by ACTIVAnalyst 5.4 software. The forward plasma power was set at $1200 \mathrm{~W}$, the Ar flow to $12 \mathrm{~L} / \mathrm{min}$, and the pump flow to $15 \mathrm{rpm}$. Measurements were performed three times per emission line, using three different elemental emission lines.

For the FTIR study, a drop of the uncured resin with and without nanoparticles was placed on a glass substrate and cured under a commercial UV-lamp (Model E0101, UV-A lamp type 3, $9 \mathrm{~W}$ ) for 6 min. Afterward, the non-polymerized resin was washed away and a thin polymerized film remained. The film was analyzed using a Perkin Elmer Spectrum Bx IR spectrometer (16 scans).

Chemicals. 1-Octadecene (technical grade 90\%, O806-1L), oleic acid (technical grade 90\%, 364525-1L), cadmium nitrate tetrahydrate $\left(\mathrm{CdNO}_{3}\left(\mathrm{H}_{2} \mathrm{O}\right)_{4}, 98 \%, 642045-100 \mathrm{G}\right)$, zinc nitrate hydrate $\left(\mathrm{ZnNO}_{3}\left(\mathrm{H}_{2} \mathrm{O}\right)\right.$, 99.999\%, 230006-25G), hydrochloric acid (puriss p.a. $\geq 37 \%$ ), nitric acid (puriss p.a., 30709-1L-GL), 1,4-dioxane 
(anhydrous, 99.8\%), 2,2'-azobis(isobutyronitrile) (AIBN, 98\%), propylene glycol methyl ether acetate (PGMEA) (99.5\%), and methyl methacrylate (contains $<30 \mathrm{ppm}$ MEHQ as the inhibitor, 99\%) were purchased from Sigma Aldrich. Cadmium acetate dihydrate $\left(\mathrm{Cd}(\mathrm{OAc})_{2}\left(\mathrm{H}_{2} \mathrm{O}\right)_{2}, 98 \%, 317131000\right)$ and sodium borohydride (99\%) were purchased from Acros Organics. Selenium powder -200 mesh (Se, 99.999\%, 36208), hydrazine monohydrate (98+\%), and 1-octylamine (99\%, B24193) were purchased from Alfa Aesar. Hexane (95\% $n$-hexane, analytical reagent grade, H/0355/21), toluene ( $\geq 99.8 \%$ ), tetrahydrofurane (THF) (analytical reagent grade, $\mathrm{T} / 070 / 17$ ), and dichloromethane (DCM) (HPLC grade) were purchased from Fisher Chemical. Chloroform, stabilized with amylene (100\%, 83627.290) and absolute ethanol (20821.330) were purchased from VWR Chemicals. Thioacetamide (TAA, >98\%, T0187) was purchased from TCI. Silver nitrate $(\geq 99 \%)$ and $\mathrm{NaOH}$ $0.01 \mathrm{M}$ were purchased from Roth. Gold acid $\left(\mathrm{HAuCl}_{4}\right.$ (99.9\%-Au)) was purchased from STREM chemicals. Tetra- $n$-octyl ammonium bromide (TBOA) (for synthesis), 2-propanol (99.5\%), and 1dodecanethiol (for synthesis) were purchased from Merck. 2Phenyl-2-propyl benzodithioate (95\%) was purchased from Acros Pharmatech Limited. The printing resins (IP-D and IP-S) were purchased from Nanoscribe. Methyl methacrylate was passed through a column of inhibitor remover prior to use. All other chemicals were used without further purification.

Thiol-Terminated Poly(methyl methacrylate) (PMMA-SH). Raft Polymerization of PMMA (PMMA-Raft). The PMMA polymer was synthesized according to a described procedure by Roth et al. ${ }^{38}$ $25 \mathrm{~g}(250 \mathrm{mmol})$ of methyl methacrylate, $1.36 \mathrm{~g}(5 \mathrm{mmol})$ of 2 phenyl-2-propyl benzodithioate, $102.5 \mathrm{mg}(0.625 \mathrm{mmol})$ of AIBN, and $25 \mathrm{~mL}$ of freshly distilled dioxane were combined in a dry Schlenk flask, the mixture was degassed by three freeze-pump-thaw cycles and then heated at $70{ }^{\circ} \mathrm{C}$ for $9 \mathrm{~h}$. The polymer (monomer conversion $=80 \% ; M_{\mathrm{n}}=4727 \mathrm{~g} / \mathrm{mol}, M_{\mathrm{w}} / M_{\mathrm{n}}=1.49$ ) was precipitated three times in cold methanol.

Hydrazinolysis (PMMA-SH). The RAFT group of the synthesized polymer was cleaved according to a slightly modified procedure by Fan et al. ${ }^{39}$ The PMMA-Raft polymer $(4.339 \mathrm{~g}, 0.92 \mathrm{mmol})$ was dissolved in dimethylformamide (DMF) $(30 \mathrm{~mL})$. The solution was degassed by three freeze-pump-thaw cycles. At RT, hydrazine monohydrate $(0.4 \mathrm{~mL}, 8.23 \mathrm{mmol})$ was added. After stirring for $2 \mathrm{~h}$ at $\mathrm{RT}$, the polymer was precipitated in excess of cold $1.2 \mathrm{M} \mathrm{HCl}$ aqueous solution. The product was filtered, washed several times with cold methanol and dried in vacuo at $45{ }^{\circ} \mathrm{C}$ overnight. The successful cleavage of the RAFT group was proven by NMR (see the SI 1).

Synthesis of Au NPs. Au NPs were synthesized by a liquid-liquid synthesis route according to a described procedure by Riedinger et al. ${ }^{40}$ Briefly, $\mathrm{HAuCl}_{4}(300 \mathrm{mg}, 0.88 \mathrm{mmol})$ was dissolved in MilliQ water $(25 \mathrm{~mL})$. Tetraoctylammoniumbromide (TOAB) $(2.17 \mathrm{~g}, 3.97$ $\mathrm{mmol})$ was dissolved in toluene $(80 \mathrm{~mL})$. Both solutions were combined in a $500 \mathrm{~mL}$ separation funnel and shaken for $5 \mathrm{~min}$. The gold precursor was transferred from the aqueous phase to the organic phase indicated by a color change from colorless to dark red. After complete separation, the aqueous solution was discarded. The organic phase was transferred to a $250 \mathrm{~mL}$ flask. Under stirring, $\mathrm{NaBH}_{4}(334$ $\mathrm{mg}, 8.83 \mathrm{mmol}$ ) dissolved in $20 \mathrm{~mL}$ of MilliQ water was added dropwise to the organic phase. A color change from red to violet could be observed due to the reduction of $\mathrm{Au}^{3+}$ to $\mathrm{Au}^{0}$. The suspension was stirred for $60 \mathrm{~min}$ and then transferred to a separation funnel. The suspension was washed once with $25 \mathrm{~mL}$ of $10 \mathrm{mM} \mathrm{HCl}$, once with $25 \mathrm{~mL}$ of $10 \mathrm{mM} \mathrm{NaOH}$, and four times with $25 \mathrm{~mL}$ of MilliQ water. The aqueous phase was discarded and the organic phase was stirred overnight.

To stabilize the nanoparticles further, either 1-dodecanethiol or a PMMA-SH polymer was used. To stabilize the nanoparticles with 1dodecanethiol, 1-dodecanethiol $(10 \mathrm{~mL}, 41.8 \mathrm{mmol})$ was added and the suspension was heated to $65{ }^{\circ} \mathrm{C}$ under stirring for $2 \mathrm{~h}$. The suspension was centrifuged at $2000 \mathrm{rpm}$ (416 rcf) for $5 \mathrm{~min}$ to remove bigger aggregates. The suspension was divided into four 50 $\mathrm{mL}$ centrifugation tubes and filled up with methanol to precipitate the NPs. The suspension was centrifuged at $2000 \mathrm{rpm}$ (416 rcf) for 5 min. The precipitate was redispersed in $8 \mathrm{~mL}$ of chloroform. To functionalize the Au NPs with the PMMA-SH polymer, $10 \mathrm{~mL}$ of the $\mathrm{Au}$ NP containing suspension was mixed with the polymer $(825 \mathrm{mg}$ ) and stirred for $30 \mathrm{~min}$ at RT. The suspension was heated up to $65^{\circ} \mathrm{C}$ under stirring for $2 \mathrm{~h}$. Then, it was stirred overnight at RT. The Au NPs were precipitated with methanol. The solution was kept in the fridge for several days and was then centrifuged at $2000 \mathrm{rpm}$ (416 rcf) for $5 \mathrm{~min}$. The precipitate was redispersed in DCM.

Synthesis of Ag NPs. Ag NPs were synthesized according to a slightly modified protocol of Niskanen et al. ${ }^{41}$ The Ag NPs were stabilized with the synthesized thiol-terminated PMMA polymer. The polymer $(0.189 \mathrm{~g}, 0.04 \mathrm{mmol})$ was dissolved in $200 \mathrm{~mL}$ of ethanol/ THF $(1: 1 \mathrm{v} / \mathrm{v}) . \mathrm{AgNO}_{3}(0.0679 \mathrm{~g}, 0.4 \mathrm{mmol})$ dissolved in $0.4 \mathrm{~mL}$ of MilliQ water and $\mathrm{NaBH}_{4}(0.152 \mathrm{~g}, 4 \mathrm{mmol})$ dissolved in $1.6 \mathrm{~mL}$ of MilliQ water were added under stirring. Immediately after the addition, hexane was added to precipitate the formed Ag NPs. The suspension was centrifuged at $4000 \mathrm{rpm}$ (1664 rcf) for $5 \mathrm{~min}$ and redispersed in DCM.

Synthesis of Core/Shell CdSe Nanoplatelets. Synthesis of CdSe NPLs. 4 monolayer (ML) thick CdSe NPLs were synthesized by a modified procedure published by Mahler et al. ${ }^{42}$ Briefly, $60 \mathrm{~mL}$ of 1 octadecene, $320 \mathrm{mg}(1.20 \mathrm{mmol})$ of $\mathrm{Cd}(\mathrm{OAc})_{2}\left(\mathrm{H}_{2} \mathrm{O}\right)_{2}$, and $879 \mu \mathrm{L}$ $(787 \mathrm{mg}, 2.79 \mathrm{mmol})$ of oleic acid were combined inside a $100 \mathrm{~mL}$ three-necked flask attached to a water-cooled condenser. The mixture was stirred and degassed under vacuum at $110^{\circ} \mathrm{C}$ for $90 \mathrm{~min}$. After stopping the vacuum line, the flask was filled with argon and $48 \mathrm{mg}$ $(0.607 \mathrm{mmol})$ of Se powder was swiftly added into the flask. Then, the temperature was set to $240{ }^{\circ} \mathrm{C}$. When the temperature in the flask reached $205{ }^{\circ} \mathrm{C}, 160 \mathrm{mg}(0.600 \mathrm{mmol})$ of $\mathrm{Cd}(\mathrm{OAc})_{2}\left(\mathrm{H}_{2} \mathrm{O}\right)_{2}$ was added into the mixture and the reaction proceeded for $15 \mathrm{~min}$ at 240 ${ }^{\circ} \mathrm{C}$. Afterward, the flask was cooled to room temperature using a water bath and $6.7 \mathrm{~mL}$ of oleic acid was injected. The mixture was centrifuged for $10 \mathrm{~min}$ at $5000 \mathrm{rpm}$ (2599 rcf), the supernatant was discarded. Finally, the precipitated NPLs were redispersed in $12 \mathrm{~mL}$ of hexane.

Synthesis of CdSe/Cd $d_{0.33} Z n_{0.67} S$ Core/Shell NPLs. The $\mathrm{Cd}_{0.33} \mathrm{Zn}_{0.67} \mathrm{~S}$ shell was synthesized using a one-pot method, modified from an existing procedure. ${ }^{42}$ Briefly, $6 \mathrm{~mL}$ of the as-synthesized $4 \mathrm{ML}$ NPLs (with an optical density at $510 \mathrm{~nm}=30$ ) was added into a 50 $\mathrm{mL}$ round bottom flask containing a stir bar. In a separate flask, 300 $\mathrm{mg}(3.99 \mathrm{mmol})$ of TAA, $3 \mathrm{~mL}(18.2 \mathrm{mmol})$ of octylamine, and $9 \mathrm{~mL}$ of chloroform were gently mixed and then sonicated in an ultrasound bath until all of the TAA dissolved. The TAA solution was added to the NPL solution while stirring. After a couple of minutes, $500 \mu \mathrm{L}$ of $\mathrm{CdNO}_{3}$ solution $\left(0.2 \mathrm{M}\right.$ in ethanol) and $1000 \mu \mathrm{L}$ of $\mathrm{ZnNO}_{3}$ solution $(0.2 \mathrm{M}$ in ethanol) were added into the mixture. The flask was sealed with a glass stopper and left to stir for $24 \mathrm{~h}$ under ambient conditions.

To be able to precipitate the NPLs, the mixture was first concentrated by evaporating a portion of the solvent using a rotary evaporator at approximately $280 \mathrm{mbar}, 40^{\circ} \mathrm{C}$ for a couple of minutes. Then, the concentrated mixture (volume approx. $15 \mathrm{~mL}$ ) was centrifuged for $10 \mathrm{~min}$ at $8000 \mathrm{rpm}(6654 \mathrm{~g})$, the supernatant was discarded, and the precipitated NPLs were resuspended in $15 \mathrm{~mL}$ of chloroform. To better stabilize the NPLs, $300 \mu \mathrm{L}$ of $\mathrm{ZnNO}_{3}$ solution $(0.2 \mathrm{M}$ in ethanol) and $600 \mu \mathrm{L}$ of oleic acid were added into the NPL dispersion while stirring and the mixture was kept under ambient conditions for a couple of days for photoluminescence recovery.

To remove the excess oleic acid introduced in this step, methyl acetate was added to the chloroform dispersion of core/shell NPLs (in the ratio of chloroform:methyl acetate $=1: 1 \mathrm{v} / \mathrm{v}$ ). The NPLs were precipitated by centrifugation and redispersed in chloroform.

Surface Functionalization of CdSe/CdZnS NPLs with PMMA-SH. To modify the surface of the NPLs, PMMA-SH $(7 \mathrm{mg})$ was mixed with the NPL dispersion $(100 \mu \mathrm{L}, 4.5 \mathrm{mg} / \mathrm{mL}$, theoretical 23000 chains $/ \mathrm{nm}^{2}$ ) and stirred for $20 \mathrm{~min}$ at RT. Afterward, the mixture was heated up to $60{ }^{\circ} \mathrm{C}$ under stirring for $1 \mathrm{~h}$. The mixture was allowed to cool down to room temperature and stirred for an additional $20 \mathrm{~min}$. Afterward, methanol was added (methanol:reaction mixture 1:2 v/v) to precipitate the NPLs. The precipitate was centrifuged at $6500 \mathrm{rpm}$ (4393 rcf) for $5 \mathrm{~min}$ and redispersed in $100 \mu \mathrm{L}$ of DCM. 
Adjustment of the Concentration of the Nanoparticle Dispersion. To determine the concentration of Au NPs and Ag NPs, ICP-OES was performed to determine the concentration of metals. Therefore, the dried NPs ( $\mathrm{Au}$ and $\mathrm{Ag}$ ) were dissolved in aqua regia and later diluted with MilliQ water, to reach a metal concentration range between 2 and $20 \mathrm{mg} / \mathrm{L}$. The concentration of the $\mathrm{Au}$ and $\mathrm{Ag}$ NP dispersions were set to $10 \mathrm{mg} / \mathrm{mL}$. The concentration of the NPL dispersion was determined by measuring the absorbance and applying a calibration curve (absorbance vs determined weight concentration by ICP). Therefore, the concentration of core/shell NPLs were measured using ICP-OES. ${ }^{43}$ The weight concentration of the NPLs in dispersion can be calculated by adding the metal ion concentration measured by ICP to $\mathrm{Se}$ and $\mathrm{S}$ concentration based on the stoichiometry

$$
\begin{aligned}
& \text { NPL concentration }\left(\frac{\mathrm{mg}}{\mathrm{mL}}\right) \\
&=\text { measured } \mathrm{Cd}^{2+} \text { concentration } \\
&+ \text { measured } \mathrm{Zn}^{2+} \text { concentration } \\
&+ \text { estimated } \mathrm{Se}^{2-} \text { concentration } \\
&+ \text { estimated } \mathrm{S}^{2-} \text { concentration }
\end{aligned}
$$

Dispersion of NPs in the Resin. The resin was diluted in DCM (1 $\mathrm{mL}$ of resin in $1 \mathrm{~mL}$ of DCM) to lower the viscosity. A certain volume of the NPs/NPL dispersion was added to reach a set final concentration (see Table 1). To facilitate the dispersion of the flat

Table 1. NPs/NPLs Dispersed in the Resin for 3D Printing in Various Concentrations

\begin{tabular}{ccc}
\hline $\begin{array}{c}\text { Ag NPs in IP-Dip } \\
(\mathrm{v} / \mathrm{v} \%)\end{array}$ & $\begin{array}{c}\text { Au NPs in IP-Dip } \\
(\mathrm{v} / \mathrm{v} \%)\end{array}$ & $\begin{array}{c}\text { CdSe/CdZnS NPLs in IP-Dip } \\
(\mathrm{v} / \mathrm{v} \%)\end{array}$ \\
0.013 & 0.02 & 0.06 \\
0.007 & 0.01 & 0.026 \\
0.0013 & 0.002 & 0.013 \\
\hline
\end{tabular}

$\mathrm{CdSe} / \mathrm{CdZnS}$ NPLs, the dispersion was diluted and the resin was slightly heated. After mixing using a vortex mixer, the solvents were removed using a rotary evaporator. The prepared resins were stored in the dark at $5{ }^{\circ} \mathrm{C}$ in the fridge.

Printing with a Nanoscribe $3 D$ Printer. The structures printed were pyramids and woodpile structures. All were fabricated using a NanoScribe Photonic Professional (GT) 3D-printer. To start printing a desired structure, it is needed to either convert a 3D-model (in. stl format) into a printer-readable format or directly program a printerreadable file. Both can be done using software DeScribe, which is supplied by NanoScribe $\mathrm{GmbH}$. The pyramids were processed from an already existing. stl file, whereas the woodpile structures were directly programmed. The woodpile structure is composed of 10 stacked layers. Each layer has 15 consecutive parallel lines, each $1 \mu \mathrm{m}$ apart from each other, and each consecutive layer is rotated by $90^{\circ}$, creating a grid. The pyramid array consists of nine square pyramids, each having a dimension of $10 \mu \mathrm{m} \times 10 \mu \mathrm{m} \times 5 \mu \mathrm{m}$. The structures were printed inside a droplet of negative photoresin (IP-Dip) using dip-in laser lithography (DiLL), where the objective is immersed directly onto the photoresin. The $63 \times$ N.A 1.4 immersion objective and $700 \mu \mathrm{m}$ thick fused silica slide as the substrate was used. Afterward, the excess photoresin was rinsed off of the structure via immersion in propylene glycol methyl ether acetate (PGMEA) for 30 $\mathrm{min}$, followed by immersion in isopropanol for $5 \mathrm{~min}$ and gentle drying with nitrogen flow. Depending on the structure, shape, and size, different parameters must be used for printing: the laser power (LP) determines the size of the individual voxels that compose the structure, which in turn determines the minimum in-plane distance each printed line must have. If the distance between the lines is too short, bubbles appear on the printed area due to local overheating, ${ }^{44}$ destroying the sample. If the distance is too large, the lines are unconnected. This is desirable in the in-plane direction for the woodpile structure, but not for the "continuous" pyramid. On the vertical axis, on both cases connected voxels are desired. If they are not properly connected, the voxels will float on the uncured resin and be washed away alongside the excess resin during the development process. On the continuous structures, the in-plane line distance is governed by the hatching distance (HD) parameter, which gives the distance in micrometers that the center of two consecutive lines will be spaced apart. The vertical distance between two voxels is controlled by the slicing distance (SD) parameter, whereas for the woodpile and grating structures this is controlled by the z-offset parameter. Since the voxel size not only depends on the LP, but also on the photoresin and NP loading, a parameter sweep of not only voxel distances, but also LP is needed for each NP-doped photoresin.

\section{ASSOCIATED CONTENT}

\section{Supporting Information}

The Supporting Information is available free of charge at https://pubs.acs.org/doi/10.1021/acsami.0c14546.

General scheme of TPP 3D printing; NMR of the PMMA polymer; further characterization of modified nanoparticles (TEM and spectroscopy); further characterization of printed objects (SEM, TEM, EDX, and IR); estimations of the interparticle distance; diffusion length and voxel volume; characterization of the photoresin (GPC, NMR) (PDF)

\section{AUTHOR INFORMATION}

\section{Corresponding Authors}

Katharina Landfester - Max Planck Institute for Polymer Research, 55128 Mainz, Germany; 이이.org/0000-00019591-4638; Email: landfester@mpip-mainz.mpg.de

Andreas Riedinger - Max Planck Institute for Polymer Research, 55128 Mainz, Germany; 이 orcid.org/0000-00027732-0606; Email: riedinger@mpip-mainz.mpg.de

\section{Authors}

Rebecca Momper - Max Planck Institute for Polymer Research, 55128 Mainz, Germany

Antonio Ibanez Landeta - Max Planck Institute for Dynamics and Self-Organization, 37077 Göttingen, Germany

Long Yang - Max Planck Institute for Polymer Research, 55128 Mainz, Germany

Henry Halim - Max Planck Institute for Polymer Research, 55128 Mainz, Germany

Heloise Therien-Aubin - Max Planck Institute for Polymer Research, 55128 Mainz, Germany; 이이.org/0000-00034567-516X

Eberhard Bodenschatz - Max Planck Institute for Dynamics and Self-Organization, 37077 Göttingen, Germany

Complete contact information is available at:

https://pubs.acs.org/10.1021/acsami.0c14546

\section{Author Contributions}

R.M., A.R., and K.L. conceived the project. Syntheses were performed by R.M., L.Y., and H.H. 3D printing was performed by R.M. and A.I.L. Characterization of 3D printed structures were performed by R.M., A.R., and K.L. wrote the manuscript. All authors contributed to the discussion of the results and to the revision of the manuscript.

\section{Notes}

The authors declare no competing financial interest. 


\section{ACKNOWLEDGMENTS}

The authors are grateful for the financial support of the Max Planck Center for Complex Fluid Dynamics. H.H. was supported by a fellowship from the Max Planck Graduate Center (MPGC), Mainz, Germany. We thank Gunnar Glasser and Katrin Kirchhoff for help with electron microscopy, Maren Müller for the preparation of the TEM lamella, and Anke Kaltbeitzel and Andreas Best for help with optical microscopy.

\section{REFERENCES}

(1) Manapat, J. Z.; Chen, Q.; Ye, P.; Advincula, R. C. 3D Printing of Polymer Nanocomposites via Stereolithography. Macromol. Mater. Eng. 2017, 302, No. 1600553.

(2) Sugioka, K.; Cheng, Y. Femtosecond Laser Three-Dimensional Micro- and Nanofabrication. Appl. Phys. Rev. 2014, 1, No. 041303.

(3) Straub, M.; Nguyen, L. H.; Fazlic, A.; Gu, M. Complex-shaped Three-Dimensional Microstructures and Photonic Crystals Generated in a Polysiloxane Polymer by Two-Photon Microstereolithography. Opt. Mater. 2004, 27, 359-364.

(4) Malinauskas, M.; Žukauskas, A.; Purlys, V.; Gaidukevičiūtè, A.; Balevičius, Z.; Piskarskas, A.; Fotakis, C.; Pissadakis, S.; Gray, D.; Gadonas, R.; Vamvakaki, M.; Farsari, M. 3D Microoptical Elements formed in a Photostructurable Germanium Silicate by Direct Laser Writing. Opt. Lasers Eng. 2012, 50, 1785-1788.

(5) Schizas, C.; Melissinaki, V.; Gaidukeviciute, A.; Reinhardt, C.; Ohrt, C.; Dedoussis, V.; Chichkov, B. N.; Fotakis, C.; Farsari, M.; Karalekas, D. On the Design and Fabrication by Two-Photon Polymerization of a readily Assembled Micro-Valve. Int. J. Adv. Manuf. Technol. 2010, 48, 435-441.

(6) Danilevicius, P.; Rezende, R. A.; Pereira, F. D. A. S.; Selimis, A.; Kasyanov, V.; Noritomi, P. Y.; Silva, J. V. L. d.; Chatzinikolaidou, M.; Farsari, M.; Mironov, V. Burr-like, laser-made 3D Microscaffolds for Tissue Spheroid Encagement. Biointerphases 2015, 10, No. 021011.

(7) Sakellari, I.; Kabouraki, E.; Gray, D.; Purlys, V.; Fotakis, C.; Pikulin, A.; Bityurin, N.; Vamvakaki, M.; Farsari, M. DiffusionAssisted High-Resolution Direct Femtosecond Laser Writing. ACS Nano 2012, 6, 2302-2311.

(8) Ovsianikov, A.; Chichkov, B. N. Two-Photon PolymerizationHigh Resolution 3D Laser Technology and Its Applications. In Nanoelectronics and Photonics: From Atoms to Materials, Devices, and Architectures; Korkin, A.; Rosei, F., Eds.; Springer New York: New York, NY, 2008; pp. 427-446.

(9) Carlotti, M.; Mattoli, V. Functional Materials for Two-Photon Polymerization in Microfabrication. Small 2019, 15, No. 1902687.

(10) Leigh, S. J.; Bradley, R. J.; Purssell, C. P.; Billson, D. R.; Hutchins, D. A. A Simple, Low-Cost Conductive Composite Material for 3D Printing of Electronic Sensors. PLoS One 2012, 7, No. e49365.

(11) Ceylan, H.; Yasa, I. C.; Yasa, O.; Tabak, A. F.; Giltinan, J.; Sitti, M. 3D-Printed Biodegradable Microswimmer for Theranostic Cargo Delivery and Release. ACS Nano 2019, 13, 3353-3362.

(12) Marino, A.; Barsotti, J.; de Vito, G.; Filippeschi, C.; Mazzolai, B.; Piazza, V.; Labardi, M.; Mattoli, V.; Ciofani, G. Two-Photon Lithography of 3D Nanocomposite Piezoelectric Scaffolds for Cell Stimulation. ACS Appl. Mater. Interfaces 2015, 7, 25574-25579.

(13) Farahani, R. D.; Dubé, M.; Therriault, D. Three-Dimensional Printing of Multifunctional Nanocomposites: Manufacturing Techniques and Applications. Adv. Mater. 2016, 28, 5794-5821.

(14) Jonušauskas, L.; Lau, M.; Gruber, P.; Gökce, B.; Barcikowski, S.; Malinauskas, M.; Ovsianikov, A. Plasmon assisted 3D Microstructuring of Gold Nanoparticle-doped Polymers. Nanotechnology 2016, 27, No. 154001.

(15) Jonušauskas, L.; Varapnickas, S.; Rimšelis, G.; Malinauskas, M. In Plasmonically Enhanced 3D Laser Lithography for High-Throughput Nanoprecision Fabrication, SPIE LASE San Francisco, 2017.

(16) Ueno, K.; Juodkazis, S.; Shibuya, T.; Mizeikis, V.; Yokota, Y.; Misawa, H. Nanoparticle-Enhanced Photopolymerization. J. Phys. Chem. C 2009, 113, 11720-11724.
(17) Murazawa, N.; Ueno, K.; Mizeikis, V.; Juodkazis, S.; Misawa, H. Spatially Selective Nonlinear Photopolymerization Induced by the Near-Field of Surface Plasmons Localized on Rectangular Gold Nanorods. J. Phys. Chem. C 2009, 113, 1147-1149.

(18) Peng, Y.; Jradi, S.; Yang, X.; Dupont, M.; Hamie, F.; Dinh, X. Q.; Sun, X. W.; Xu, T.; Bachelot, R. 3D Photoluminescent Nanostructures Containing Quantum Dots Fabricated by TwoPhoton Polymerization: Influence of Quantum Dots on the Spatial Resolution of Laser Writing. Adv. Mater. Technol. 2019, 4, No. 1800522.

(19) Liu, Y.; Xiong, W.; Li, D. W.; Lu, Y.; Huang, X.; Liu, H.; Fan, L. S.; Jiang, L.; Silvain, J.-F.; Lu, Y. F. Precise Assembly and Joining of Silver Nanowires in Three Dimensions for highly conductive Composite Structures. Int. J. Extreme Manuf. 2019, 1, No. 025001.

(20) Bhushan, B.; Kundu, T.; Singh, B. P. Two-photon Absorption Spectrum of Silver Nanoparticles. Opt. Commun. 2012, 285, 54205424.

(21) Scott, R.; Achtstein, A. W.; Prudnikau, A.; Antanovich, A.; Christodoulou, S.; Moreels, I.; Artemyev, M.; Woggon, U. Two Photon Absorption in II-VI Semiconductors: The Influence of Dimensionality and Size. Nano Lett. 2015, 15, 4985-4992.

(22) Olesiak-Banska, J.; Waszkielewicz, M.; Obstarczyk, P.; Samoc, M. Two-photon Absorption and Photoluminescence of Colloidal Gold Nanoparticles and Nanoclusters. Chem. Soc. Rev. 2019, 48, 4087-4117.

(23) Zhang, Z.; Berg, A.; Levanon, H.; Fessenden, R. W.; Meisel, D. On the Interactions of Free Radicals with Gold Nanoparticles. J. Am. Chem. Soc. 2003, 125, 7959-7963.

(24) Chappell, M. A.; Miller, L. F.; Price, C. L. Reversible Exchange of Stable Nitroxyl Radicals on Nanosilver Particles. Environ. Chem. 2015, 12, 198-203.

(25) Maurel, V.; Laferrière, M.; Billone, P.; Godin, R.; Scaiano, J. C. Free Radical Sensor Based on CdSe Quantum Dots with Added 4Amino-2,2,6,6-Tetramethylpiperidine Oxide Functionality. J. Phys. Chem. B 2006, 110, 16353-16358.

(26) Kumar, S. K.; Krishnamoorti, R. Nanocomposites: Structure, Phase Behavior, and Properties. Annu. Rev. Chem. Biomol. Eng. 2010, $1,37-58$.

(27) Schaefer, D. W.; Justice, R. S. How Nano Are Nanocomposites? Macromolecules 2007, 40, 8501-8517.

(28) Asakura, S.; Oosawa, F. On Interaction between Two Bodies Immersed in a Solution of Macromolecules. J. Chem. Phys. 1954, 22, $1255-1256$.

(29) Wei, Q. H.; Su, K. H.; Durant, S.; Zhang, X. Plasmon Resonance of Finite One-Dimensional Au Nanoparticle Chains. Nano Lett. 2004, 4, 1067-1071.

(30) Corbierre, M. K.; Cameron, N. S.; Sutton, M.; Laaziri, K.; Lennox, R. B. Gold Nanoparticle/Polymer Nanocomposites: Dispersion of Nanoparticles as a Function of Capping Agent Molecular Weight and Grafting Density. Langmuir 2005, 21, 60636072.

(31) Smith, G. D.; Bedrov, D. Dispersing Nanoparticles in a Polymer Matrix: Are Long, Dense Polymer Tethers Really Necessary? Langmuir 2009, 25, 11239-11243.

(32) Zhao, P. X.; Li, N.; Astruc, D. State of the Art in Gold Nanoparticle Synthesis. Coord. Chem. Rev. 2013, 257, 638-665.

(33) Battocchio, C.; Meneghini, C.; Fratoddi, I.; Venditti, I.; Russo, M. V.; Aquilanti, G.; Maurizio, C.; Bondino, F.; Matassa, R.; Rossi, M.; Mobilio, S.; Polzonetti, G. Silver Nanoparticles Stabilized with Thiols: A Close Look at the Local Chemistry and Chemical Structure. J. Phys. Chem. C 2012, 116, 19571-19578.

(34) Rogach, A. L.; Kornowski, A.; Gao, M.; Eychmüller, A.; Weller, H. Synthesis and Characterization of a Size Series of Extremely Small Thiol-Stabilized CdSe Nanocrystals. J. Phys. Chem. B 1999, 103, 3065-3069.

(35) Bagheri, A.; Jin, J. Photopolymerization in 3D Printing. ACS Appl. Polym. Mater. 2019, 1, 593-611.

(36) Govorov, A. O.; Richardson, H. H. Generating Heat with Metal Nanoparticles. Nano Today 2007, 2, 30-38. 
(37) Liu, Y.; Campbell, J. H.; Stein, O.; Jiang, L.; Hund, J.; Lu, Y. Deformation Behavior of Foam Laser Targets Fabricated by TwoPhoton Polymerization. Nanomaterials 2018, 8, 498.

(38) Roth, P. J.; Kessler, D.; Zentel, R.; Theato, P. A Method for Obtaining Defined End Groups of Polymethacrylates Prepared by the RAFT Process during Aminolysis. Macromolecules 2008, 41, 83168319.

(39) Fan, Z.; Serrano, M. K.; Schaper, A.; Agarwal, S.; Greiner, A. Polymer/Nanoparticle Hybrid Materials of Precise Dimensions by Size-Exclusive Fishing of Metal Nanoparticles. Adv. Mater. 2015, 27, 3888-3893.

(40) Riedinger, A.; Zhang, F.; Dommershausen, F.; Röcker, C.; Brandholt, S.; Nienhaus, G. U.; Koert, U.; Parak, W. J. Ratiometric Optical Sensing of Chloride Ions with Organic Fluorophore-Gold Nanoparticle Hybrids: A Systematic Study of Design Parameters and Surface Charge Effects. Small 2010, 6, 2590-2597.

(41) Niskanen, J.; Shan, J.; Tenhu, H.; Jiang, H.; Kauppinen, E.; Barranco, V.; Picó, F.; Yliniemi, K.; Kontturi, K. Synthesis of Copolymer-Stabilized Silver Nanoparticles for Coating Materials. Colloid Polym. Sci. 2010, 288, 543-553.

(42) Mahler, B.; Nadal, B.; Bouet, C.; Patriarche, G.; Dubertret, B. Core/Shell Colloidal Semiconductor Nanoplatelets. J. Am. Chem. Soc. 2012, 134, 18591-18598.

(43) Halim, H.; Simon, J.; Lieberwirth, I.; Mailaender, V.; Koynov, K.; Riedinger, A. Water-dispersed Semiconductor Nanoplatelets with high fluorescence Brightness, chemical and colloidal Stability. J. Mater. Chem. B 2020, 8, 146-154.

(44) Guney, M. G.; Fedder, G. K. Estimation of Line Dimensions in 3D Direct Laser Writing Lithography. J. Micromech. Microeng. 2016, 26, No. 105011. 